\title{
Finding a cure for sickle cell disease
}

\author{
Julie Makani is an associate professor in the Department of Haematology and Blood Transfusion at Muhimbili \\ University of Health and Allied Sciences in Dar es Salaam, where she helped Tanzania establish one of the world's \\ largest single-center study cohorts for sickle cell disease. She received the 2011 Royal Society Africa Award on the \\ translation of genomics to health benefits.
}

\section{Julie Makani}

n 2003, I made a decision to conduct research on sickle cell disease (SCD). My decision was met with concern, as the major focus at the time in Tanzania, as in many other countries in Africa, was addressing the health burden due to infections such as malaria, tuberculosis and HIV/AIDS. It was difficult for people to see the value of research on a genetic condition in Africa, which was considered to be rare because "many people died anyway" and for which, even if they survived, there was no treatment.

I, however, have personally witnessed the excruciating painful episodes known as vaso-occlusive crises that result from sickled red blood cells obstructing circulation, and I have lost family members to this disease. I believe that through research, we will be able to improve the health of individuals with SCD. I was able to establish partnerships, and we started working on SCD and demonstrated the burden of disease and the unmet need. We set up a systematic framework for research and established one of the largest single-center SCD cohorts in the world, with prospective surveillance (2004-2016) of over 5,000 SCD patients. We are using this experience and data in an African initiative, SickleInAfrica, that consists of three consortia: the Sickle PanAfrica Research Consortium, Sickle Africa Data Coordinating Centre and Sickle PanAfrican Network.

In 2008, I participated in developing a strategy for genomic research, Human Heredity and Health in Africa (H3Africa). This was considered ambitious, and again concerns were raised about the value of genomic research in Africa. The need for research in Africa was eloquently articulated, however, by the cardiologist Bongani Mayosi, who said that it is imperative to have a cadre of Africans who are highly trained and globally competitive. His shocking death in 2018 was a moment that made us recognize the intense pressure on individuals in the scientific environment and the immense responsibility of achieving the goal of conducting globally competitive research in Africa.

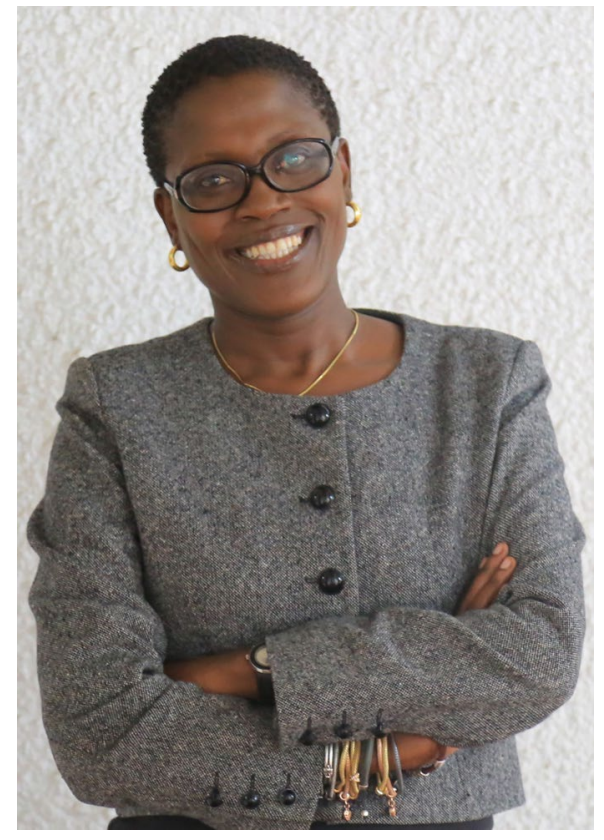

Credit: Ezekiel Kamwaga

In my research, I identified anemia as a major contributing factor to illness and death in SCD, and in 2011 I received the Royal Society Africa Award for my work as an example of the translation of genomic research into health benefits. I provided a detailed description of the epidemiology and phenotype of SCD anemia, and started looking for genetic factors that could explain the marked heterogeneity of this anemia. The initial focus was on phenotyping fetal hemoglobin, a genetically determined trait that influences disease severity and that had not been previously studied in Africa because of the difficulty of measuring the levels of this protein. Following this, we were able to conduct one of the first genome-wide association studies of fetal hemoglobin in SCD in Africa, reporting important differences from findings obtained in SCD populations outside Africa. It is known that hydroxyurea increases the level of fetal hemoglobin, but there is limited knowledge on how hydroxyurea affects hematopoiesis, the production of blood cells and subsequently anemia. Understanding this process, and the associated genetic and environmental factors, could lead to functional studies on the mechanisms of hematopoiesis and anemia. This "genetic knowledge" would allow the development of targets for the treatment of anemia and hopefully a cure for SCD.

A recent turning point in my life came when I watched a video of Nobel Laureate and virologist Françoise Barré-Sinoussi, in which she was asked why she was pushing for HIV cure research. Her response was, "Don't ask me, ask people living with HIV." This resonated with me because I and my fellow researchers faced similar skepticism when we started talking about a strategy for curative therapy for SCD in Africa. Hematopoietic stem cell transplantation can cure SCD, and there has been significant progress in improving outcomes and reducing associated complications. Even more compelling is the progress in genomics, with a 2017 report of the first successful cure of SCD using gene therapy (https://www.nejm.org/doi/ full/10.1056/NEJMoa1609677). There is justifiable concern that curative therapy for SCD is ambitious and unrealistic for Africa, if it is done in isolation. It is imperative that this strategy be accompanied by ensuring that everyone has access to basic SCD healthcare. However, there is every reason to tell individuals with SCD in Africa who ask about curative options that we are working to improve access to diagnosis and treatment, as well as conducting research to advance healthcare and ultimately cure the disease.

Julie Makani ${ }^{1,2,3,4}$

${ }^{1}$ Muhimbili University of Health and Allied Sciences (MUHAS), Dar-es-Salaam, Tanzania. ${ }^{2}$ Muhimbili National Hospital, Dar-es-Salaam, Tanzania. ${ }^{3}$ SickleInAfrica, Dar-es-Salaam, Tanzania, and Cape Town, South Africa. ${ }^{4}$ SickleGenAfrica, Accra, Ghana. e-mail:jmakani@blood.ac.tz

Published online: 5 December 2019 https://doi.org/10.1038/s41591-019-0691-0 